\title{
Orientando para facilitar el cambio en las familias: Un modelo de intervención
}

\author{
Counseling for Facilitating Changes in families: A Model for Intervention
}

Orientação para facilitar a mudança nas famílias: um modelo de intervenção

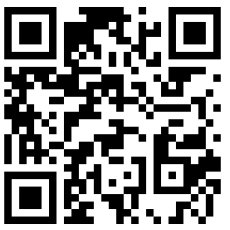

Cecilia Villarreal-Montoya

Universidad Nacional

Heredia, Costa Rica

ceciliavillarrealm@gmail.com

iD https://orcid.org/0000-0002-4623-4166

Ana Lucía Villalobos-Cordero

Universidad Nacional

Heredia, Costa Rica

avil@una.cr

https://orcid.org/0000-0002-5018-3867

Ruth Villanueva-Barbarán

Universidad Nacional

Heredia, Costa Rica

rvillanu@una.cr

https://orcid.org/0000-0001-5480-8564

Recibido • Received • Recebido: 25 / 07 / 2018

Corregido • Revised • Revisado: 06 / 12 / 2019

Aceptado • Accepted • Aprovado: 18/02 / 2020

\begin{abstract}
Resumen:La educación familiar requiere claridad epistemológica que fundamentela práctica.Trabajar colectivamente con grupos familiares es un recurso significativo de aprendizaje para potenciar sus fortalezas. El artículo presenta la sistematización de un modelo para la intervención en orientación familiar, consolidado en el Proyecto de extensión Orientando Familias (POF), Universidad Nacional. Su propósito es fortalecer habilidades de facilitación con bases teórico-metodológicas sistémicas en profesionales de diversas áreas, para que planifiquen acciones preventivas, dirigidas a mejorar la calidad de relaciones en la vida de las familias. Como insumo, se utilizaron los informes, crónicas, videos, fotografías, evaluaciones orales de cada una de las sesiones ejecutadas. Se implementa en escuelas; Hospital Nacional de Niños; Ministerio de Justicia y Paz, y CEN-CINAI de la GAM, Limón, Sarapiquí, Guanacaste, Pérez Zeledón y Puntarenas. Se capacitaron 59 personas, ejecutaron 26 planes de educación y 345 personas y sus familias beneficiadas. Las personas facilitadoras lograron reconceptualizar a las familias desde la convivencia relacional y de la construcción de vínculos, el reencuentro con la propia familia, mayor conocimiento sobre la dinámica familiar, generary desarrollar proyectos propios, efectuar una intervención más relacional para facilitar el cambio. La intervención metodológica sistémica permite a profesionales proveer a las familias de experiencias de aprendizaje
\end{abstract}


http://doi.org/10.15359/ree.24-2.9

ROR: https://ror.org/01t466c14 Universidad Nacional, Costa Rica

http://www.una.ac.cr/educare

educare@una.cr

con significación personal, que promueva oportunidades para trabajar en la transformación del sistema familiar y favorezca mejores formas de convivencia social.

Palabras claves: Orientación familiar; facilitadores; modelo de capacitación; educación familiar.

Abstract: Family education requires epistemological clarity to provide a basis for praxis. Working collectively with family groups is a significant learning resource to enhance their strengths. The article presents the systematization of a model for intervention in family counseling, consolidated in the university extension project titled "Orientando Familias" (POF, acronym in Spanish) by the Universidad Nacional de Costa Rica. Its purpose is to strengthen facilitation skills with systemic theoretical and methodological bases in professionals of diverse areas so that they can plan preventive actions aimed at improving the quality of relationships in families. Reports, chronicles, videos, photographs, and oral evaluations of each of the sessions conducted were used as inputs. The project was implemented in schools, the National Children's Hospital, the Ministry of Justice and Peace, and in the CEN-CINAIs of the Metropolitan Area of San José, Limón, Sarapiquí, Guanacaste, Pérez Zeledón, and Puntarenas. Fifty-nine people were trained, twenty-six education plans were created, and 345 people and their families were benefited. The facilitators were able to reconceptualize the families from the relational coexistence and the construction of bonds, the reunion with the family itself, greater knowledge about family dynamics, generation and developing of own projects, and staging a more relational intervention to facilitate change. The systemic methodological intervention allowed the professionals to provide families with learning experiences and personal significance promoting opportunities to work in the transformation of the family system and favor better forms of social coexistence.

Keywords: Family counseling; facilitators; training model; family education.

Resumo: A educação familiar requer clareza epistemológica que fundamente a prática. Trabalhar com gruposfamiliares éum recurso significativo de aprendizagem para potenciar seus pontos fortes. $O$ artigo apresenta a sistematização de um modelo para a intervenção em Orientação Familiar, consolidado no Projeto de Extensão"Orientando Famílias" (POF), da Universidade Nacional de Costa Rica. Seu propósito é fortalecer habilidades de facilitação com bases teórico-metodológicas sistêmicas em profissionais diversos, para que planejem ações preventivas, dirigidas a melhorar a qualidade de relações na vida das famílias. Como insumo, foram utilizados os relatórios, crônicas, vídeos, fotografias, avaliações orais de cada uma das sessões executadas. É implementado em escolas, Hospital das criancas, Ministério de Justiça e Paz, CEN-CINAl, de GAM (Grande área Metropolitana), Limón, Sarapiquí, Guanacaste, Pérez Zeledón e Puntarenas. Foram treinadas 59 pessoas, executados 26 planos educacionais sendo que 345 pessoas e suas famílias foram beneficiadas. As pessoas facilitadoras conseguem elaborar um novo conceito as famílias desde a convivência relacional e da construção de vínculos, o reencontro com a própria família, maior conhecimento sobre a dinâmica familiar, gerar e desenvolver projetos próprios, efetuar uma intervenção mais relacional permitindo facilitar para a mudança. A intervenção metodológica sistêmica permite a profissionais prover às famílias de experiências de aprendizagem com significado pessoal, que promova oportunidades para trabalhar na transformação do sistema familiar e promova melhores formas de convivência social.

Palavras chaves: Orientação familiar, facilitadores, modelo de treinamento, educação familiar. 
http://doi.org/10.15359/ree.24-2.9

ROR: https://ror.org/01t466c14 Universidad Nacional, Costa Rica http://www.una.ac.cr/educare educare@una.cr

\section{Introducción}

El artículo presenta la sistematización de experiencias de una investigación-acción de un modelo de formación teórico-metodológica para la intervención en orientación familiar, que se ha venido consolidando en el Proyecto de extensión: Orientando Familias (POF), de la División de Educación para el Trabajo, Centro de Investigación y Docencia en Educación (CIDE), Universidad Nacional de Costa Rica (UNA). Este fue concebido y desarrollado para la capacitación de equipos interdisciplinarios (integrados por profesionales en orientación, administración, docencia, ciencias sociales y de salud), de los Ministerios de Educación, de Justicia y Paz y de Salud, Hospital Nacional de Niños y CEN-CINAI, que provenían inicialmente de Alajuela, Heredia y San José, luego se amplió la cobertura al resto de las provincias del país. Estas capacitaciones se dieron como respuesta a la necesidad, percibida en las familias, de apoyo para ejercer sus funciones de crianza. Desde este panorama, se propone como problema de investigación: Cómo fortalecer habilidades de facilitación en profesionales que trabajan con familias para que brinden apoyo desde una cosmovisión sistémica.

Estas capacitaciones fueron lideradas por un equipo capacitador ${ }^{1}$ integrado por orientadoras y estudiantes asistentes de esta misma disciplina (Equipo POF).

Su propósito principal fue fortalecer habilidades de facilitación de profesionales, fundamentadas en criterios ecológico-sistémicos. Los propósitos específicos fueron:

a. Comprender, con una mirada sistémica, la convivencia familiar.

b. Construir planeamientos de intervención familiar sistémica.

c. Desarrollar planes de educación para las familias.

Las experiencias tuvieron como propósito fortalecer habilidades de facilitación de profesionales, fundamentadas en criterios ecológico-sistémicos, para dar sentido y pertinencia a la planificación de acciones dirigidas a mejorar la calidad de relaciones en la vida de las familias que conformaban los diferentes centros.

En este modelo, las personas participantes primero se posicionaron como integrantes de un sistema familiar; vivenciaron la metodología sistémica, la cual es basada en el enfoque sistémico que destaca el pensamiento circular que resalta los vínculos entre los diferentes sistemas y subsistemas (Fuhrmann y Chadwick, 1998; O'Connor y McDermott, 1998; Vallejo Valencia, 2008). De esta forma, al comprender su propia familia y apropiándose de destrezas metodológicas, asumieron un rol facilitador; implementaron proyectos (planes de educación

${ }^{1}$ Cecilia Villarreal Montoya, Ruth Villanueva Barbarán, Ana Lucía Villalobos Cordero, Roxana Chinchilla Jiménez. 
http://doi.org/10.15359/ree.24-2.9

ROR: https://ror.org/01t466c14 Universidad Nacional, Costa Rica

http://www.una.ac.cr/educare

educare@una.cr

a las familias) dirigidos a madres, padres y otras personas responsables de la niñez de comunidades a las que brindaban servicios, algunas inmersas en contextos vulnerables de pobreza. Este modelo propone, dentro de su metodología, un proceso de orientación por etapas, la implementación de un rol facilitador (aprendiz-facilitar, cambiar-facilitar, multiplicarmultiplicar) y un planeamiento circular, en donde todos los elementos se vinculan.

\section{Antecedentes}

Indagaciones muestran que, cuando la crianza no se cumple adecuadamente, las familias expresan dificultades relacionales; baja cohesión, poco afecto, inadecuado manejo de límites (Prieto Cruz, 2015) y de comunicación (Ministerio de Salud, OPS, 2004) que facilitan el uso de la violencia como práctica educativa. Estos problemas de convivencia se muestran en aumento de divorcios (Marín, 2018), violencia infantil como epidemia (Sibaja, 2018; Villagra, 2011), deserción escolar, problemas disciplinarios, consumo riesgoso de alcohol (IAFA, 2011), explotación diversa, robo, mendicidad; situaciones que son reforzadas en las familias junto al contexto social de exclusión (UCR y UNICEF, 2012).

EI POF ha realizado esfuerzos importantes en el marco de la orientación familiar, cuyas experiencias y alcances en su trayectoria posicionan a esta disciplina educativa, con liderazgo para la formación de equipos interdisciplinarios que apoyan la acción educativa que han de cumplir las familias. En años previos (2002-2007), el POF que se denominaba “Fortalecimiento de la familia" asumió la intervención directa (Escuelas para padres) mediante talleres y conferencias, con familias de escuelas vecinas al recinto universitario en Heredia. Esta experiencia generó aprendizajes para aumentar la cobertura y proyección, de modo que a partir del 2008 se incursionó en la modalidad de agentes multiplicadores, en la que se capacitó, en su mayoría a docentes para que replicaran la vivencia (Calderón Monge et al., 2009; Villarreal Montoya et al., 2014). Ambas prácticas fueron insumos para valorar resultados y mejorar la integración del enfoque teórico y la metodología en recientes experiencias del proyecto, bajo su l y ll formulación nombrada "Orientando Familias" (2013-2016).

En este recorrido, el equipo capacitador, en constante estudio y realimentación, se apropia y mantiene entre sus estrategias, priorizar la participación que surge de las vivencias de madres y padres, en vez de las charlas impartidas por profesionales (Villarreal Montoya et al., 2014). En esta misma línea, como parte de los principios teórico-metodológicos identificados y que se presentan más adelante, se siguió implementando lo que en su momento de reflexión fueron "ideas ... para favorecer la intervención grupal" (Villarreal Montoya et al., 2014, p. 235), a saber: aprender-facilitar, cambiar-facilitar y multiplicar-multiplicar.

Los alcances específicos se visualizaron en dos capacitaciones designadas "Creciendo en familia" $y$ "Fortaleciendo familias en prácticas de parentaje", cada una de ellas se organizó en dos 
periodos, en el primero se vivenciaron principios teórico-metodológicos del enfoque sistémico $y$, en el segundo, se planearon y ejecutaron planes de educación a las familias.

\section{Metodología}

Esta experiencia se realizó en el marco de la investigación-acción, la cual es un tipo de estudio dentro del enfoque cualitativo. La investigación-acción se caracteriza por reconocer los saberes populares y la capacidad inherente de afrontar las propias situaciones. En su desarrollo, logra producir conocimiento que inicia en la identificación de necesidades y culmina en la propuesta de soluciones teóricas y su aplicación (Bautista, 2011). Su metodología incluye momentos que "son flexibles ya que permiten abordar los hechos sociales como dinámicos y cambiantes, por lo tanto, están sujetos a los cambios que el mismo proceso genere" (Bautista, 2011, p. 97).

En el caso de esta experiencia, en el primer momento se indagó sobre la situación de las familias en Costa Rica, mediante investigaciones y los resultados del trabajo previo en el POF. Basado en ello, se inicia el segundo momento en el que se priorizaron las necesidades, desde las cuales se podían generar aportes desde la disciplina y proponer un modelo de orientación familiar. En un tercer momento se ejecutó la propuesta con miras a lograr una transformación social con las poblaciones beneficiadas, acompañada de una evaluación constante.

\section{Principios teórico-metodológicos del modelo, que guiaron la experiencia práctica}

La disciplina de orientación busca favorecer el desarrollo humano individual, así como el de las familias, el nicho vital primario donde las personas se forman; por ello, la propuesta de capacitación desarrollada por el POF se enmarcó, desde su formulación, en la orientación familiar, cuyo propósito educativo se enfocó en el asesoramiento sobre la comprensión de las interacciones que se dan a lo interno de las familias. En este contexto se estimuló a madres, padres, profesionales y otras personas en sus distintos roles dentro de la familia, a explorar, comprender y analizar resultados de los estilos que vivenciaron $y$, a la vez, que han utilizado como guía para la crianza. Con ello se dio la oportunidad de entender cómo se han formado las interacciones a lo interno de las familias y cuáles han sido los desaciertos y logros alcanzados. Con este ejercicio se generan posibilidades para hacer cambios, al comprender que, además del amor, son necesarios los espacios formativos para complementar la educación familiar.

La modalidad de intervención familiar que sustenta la metodología utilizada integra la intervención sistémica y la orientadora (Bisquerra Alzina y Álvarez González, 2011). La primera busca favorecer el cambio en plena vivencia, promueve el descubrimiento de conductas en la acción e interacción de la dinámica familiar. Por su parte, la intervención orientadora propicia la 
http://doi.org/10.15359/ree.24-2.9

ROR: https://ror.org/01t466c14 Universidad Nacional, Costa Rica

http://www.una.ac.cr/educare

educare@una.cr

identificación de competencias y recursos, y estimula la búsqueda de información para tomar decisiones y asumir, responsablemente, las consecuencias de estas. A partir de esta articulación se plantean tres formas de abordaje:

1. De desarrollo, en la que se coordina con instituciones, proyectos nacionales y comunitarios que apoyan las familias.

2. De capacitación o prevención: está centrada en la preparación o formación a la familia, con el fin de favorecer su dinámica interna, para que pueda administrar sus propios recursos que les faciliten mejorar el propio funcionamiento.

3. De modificación: es exclusiva para que las familias con carencias significativas busquen ayuda para manejar sus dificultades.

El trabajo realizado por el POF se enmarca dentro de la capacitación o prevención, mediante un modelo metodológico de intervención que está conformado por varios componentes interrelacionados entre sí (Figura 1). Cabe señalar que en la experiencia realizada se entendió por modelo de intervención a: "una representación que refleja el diseño, la estructura y los componentes esenciales de un proceso de intervención en orientación. ... que sirven de guía para la acción: su función consiste en proponer líneas de actuación práctica". (Bisquerra Alzina y Álvarez González, 2011, p. 55).

Su construcción tiene como fundamento la comprensión de las familias desde el enfoque sistémico, cuyos principios, aunados a los de la disciplina, son la base para desarrollar los procesos de orientación familiar, de los cuales se derivan los planeamientos circulares (elaborados por el equipo capacitador en busca de coherencia entre la teoría y la práctica sistémica) necesarios para guiar la ejecución de cada proyecto de educación a las familias, a cargo de profesionales en diversas instituciones. En el rol facilitador de las profesionales y los profesionales en mención, se implementan los principios de intervención grupal: aprender-facilitar (modestia de sentirse aprendiz, cuestionando lo aprendido para mantenerlo, deconstruirlo y reconstruirlo); cambiarfacilitar (disposición para explorar y adquirir nuevas estrategias metodológicas que conduzcan al cambio) y multiplicar-multiplicar (cuando al compartir con otras personas se multiplica y se crece) (Villarreal Montoya et al., 2014).

El iniciar el modelo metodológico con el reencuentro de la persona facilitadora con su propia familia implica una diferencia en sus habilidades de facilitación con las otras familias, pues no pretende resolver, sino facilitar, con base en el supuesto de que cada sistema familiar cuenta con sus propias fortalezas que durante el proceso orientador debe reconocer. Esto representa una gran oportunidad para las familias para fortalecer sus vínculos, pues no se encuentra en un papel receptor sino de actor de sus propios cambios. 
http://doi.org/10.15359/ree.24-2.9

ROR: https://ror.org/01t466c14 Universidad Nacional, Costa Rica

http://www.una.ac.cr/educare

Figura 1: Modelo metodológico

Modelo: Intervención en Orientación Familiar (POF)

Base teórico-metodológica con enfoque sistémico e intervención orientadora, concebida y desarrollada para la capacitación de equipos interdisciplinarios, enfocada en fortalecer habilidades de facilitación dando sentido y pertinencia a la planificación de acciones educativas para mejorar la calidad de relaciones en la vida de las familias.

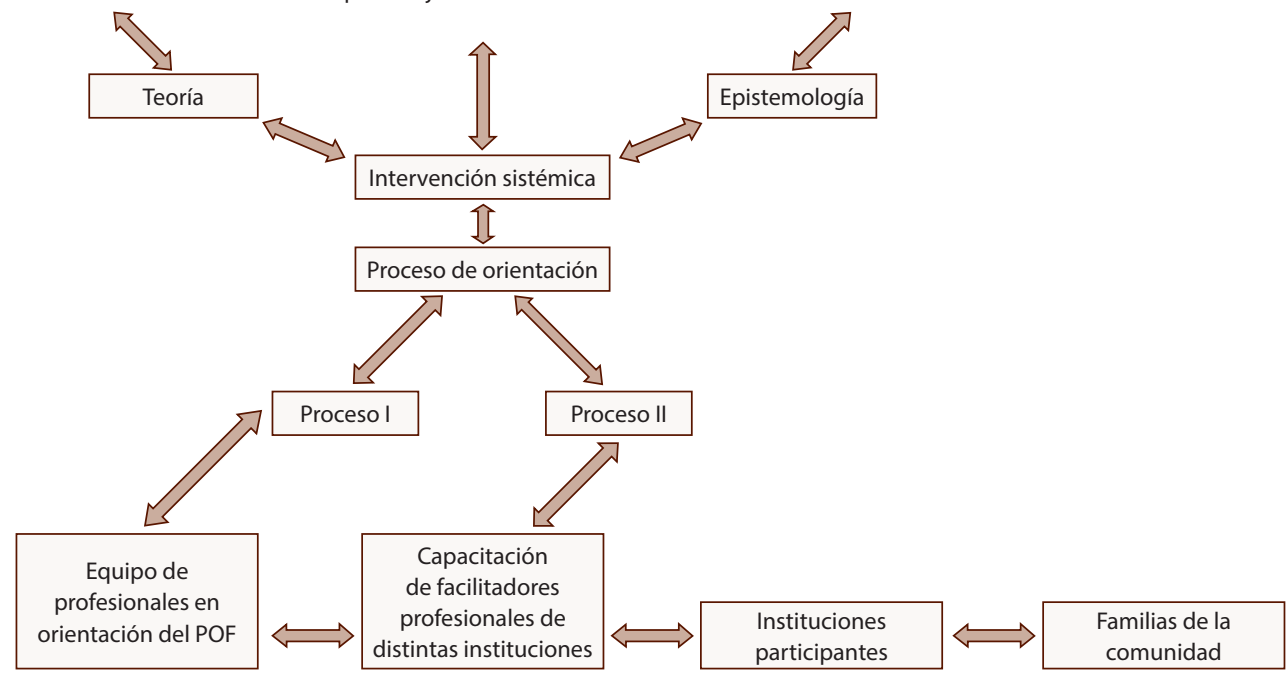

Nota: Elaboración propia.

Estos principios se han validado desde experiencias previas del POF por Villarreal Montoya et al. (2014). Finalmente, el modelo cierra y reinicia de forma cíclica con la realimentación (evaluación), a la luz de los fundamentos sistémicos. Para una mayor comprensión, obsérvese la Figura 2.

Figura 2: Proceso l: Construcción del modelo metodológico

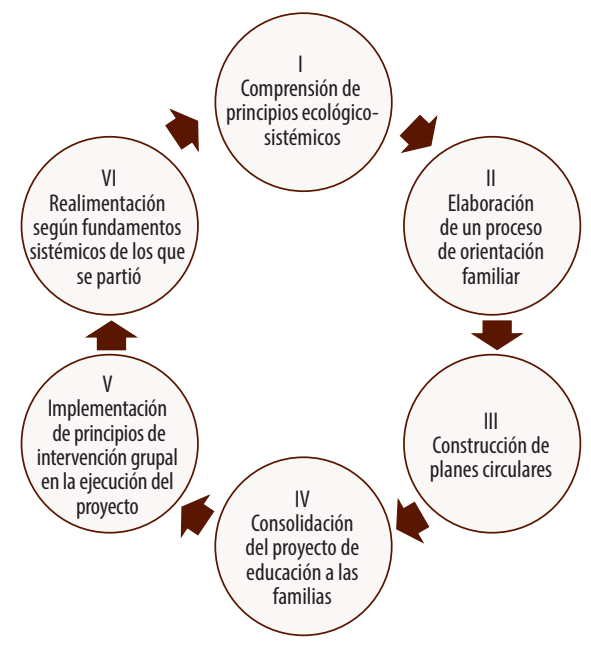

Nota: Elaboración propia 
http://doi.org/10.15359/ree.24-2.9

ROR: https://ror.org/01t466c14 Universidad Nacional, Costa Rica

http://www.una.ac.cr/educare

educare@una.cr

A continuación, se expone, de manera específica, cada uno de los componentes presentados y sus fundamentos.

\section{Proceso de orientación}

El equipo del POF se planteó un proceso I (Proceso I), base sobre el cual se ejecutó el modelo de intervención en orientación familiar. En este contexto, el proceso fue comprendido como la estructura que organiza y guía el curso del modelo en forma lógica, continua y secuencial, fundamentada en un referente teórico. Los pasos para su logro son redactados a modo de fases o etapas necesarias que están en completa relación, son enunciados cortos con los que se da a explicar, en forma sucesiva, desde dónde se debe partir y hasta dónde se requiere llegar (Figura 2).

Como parte de lo anterior, se crea el proceso II, específico de orientación familiar (Figura 3), para elaborarlo, el equipo capacitador planteó el siguiente propósito:

Vivenciar un proceso de cambio teórico, epistemológico y de intervención sistémica que permita al grupo de participantes:

a. Reencontrarse con sus propias familias y la diversidad familiar.

b. Reconceptualizar a las familias desde la convivencia relacional y de la construcción de los vínculos.

c. Familiarizarse con el tipo de planificación-intervención-valoración del cambio desde los patrones relacionales.

d. Construir un proyecto sistémico para trabajar con grupos familiares en las comunidades.

\section{Etapas}

Con base en el proceso II se organizaron tres etapas:

1. ETAPA: Una mirada sistémica de la convivencia familiar

2. ETAPA: Acercamientos al planeamiento de intervención sistémica

3. ETAPA: Realimentación de las experiencias y valoración de los cambios promovidos. 
http://doi.org/10.15359/ree.24-2.9

ROR: https://ror.org/01t466c14 Universidad Nacional, Costa Rica

http://www.una.ac.cr/educare

educare@una.cr

Figura 3: Proceso Il de intervención orientadora

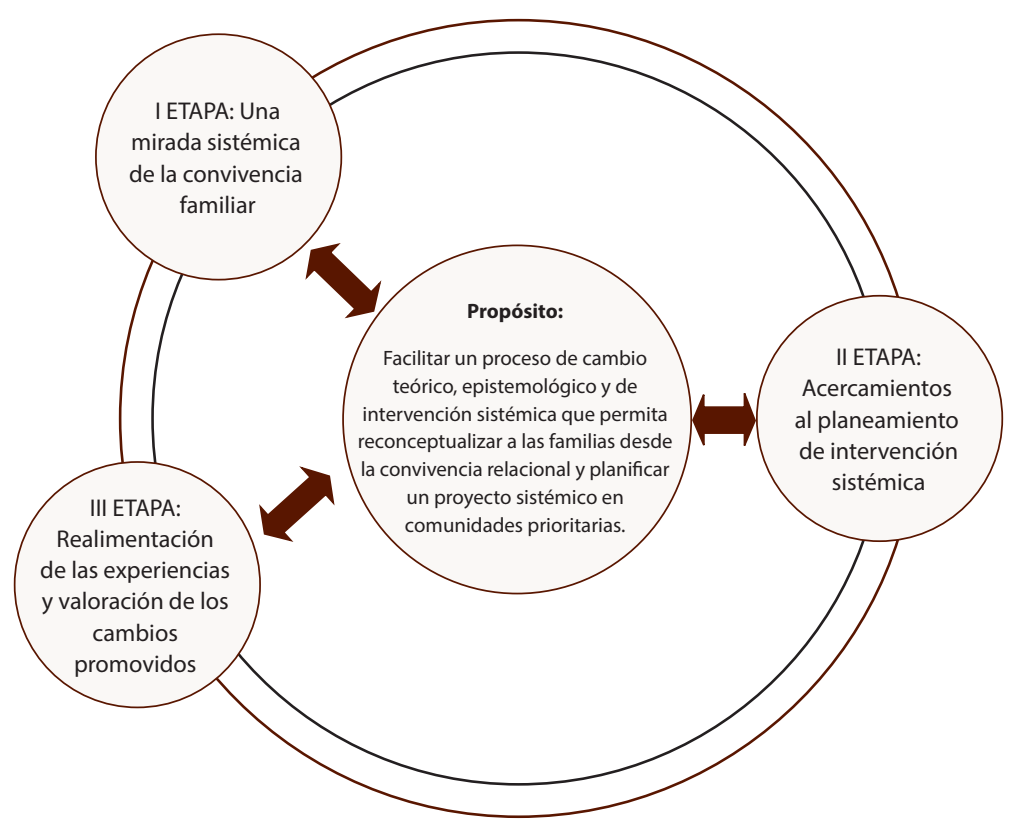

Nota: Elaboración propia.

En la práctica del modelo que se viene describiendo, la elaboración del proceso requirió del estudio constante y apropiación de los fundamentos teórico-sistémicos que guiaron la planificación, ejecución y realimentación de las capacitaciones alrededor de la comprensión del funcionamiento y diversidad de los grupos familiares. En específico, los principios que lo guiaron fueron:

\section{Las familias funcionan como sistemas}

Pese a la diversidad en la conformación de la familia, esta se concibe como un sistema cuyas relaciones entre sus miembros son circulares por lo que "se influyen mutuamente ... En este sentido una familia no es una suma de personas, ya que al formar la totalidad [adquiere sus propias cualidades]... diferentes a las que se aprecian en cada uno de sus miembros como unidades" (Arias Gallegos, 2012, p. 33). Desde esa visión ecológica-sistémica, la familia es una red de interacciones cambiantes, en constante desarrollo y de influencia recíproca con sistemas externos.

Espinal et al. (2004-2006) explican que las familias están formadas por subsistemas organizados en una jerarquía no horizontal (conyugal, formada por una pareja de personas adultas; el parental con su prole, el filial compuesto por hermanos y hermanas, y es posible que exista un subsistema extendido), estas subestructuras cumplen un rol que mantiene el funcionamiento del sistema familiar. 
http://doi.org/10.15359/ree.24-2.9

ROR: https://ror.org/01t466c14 Universidad Nacional, Costa Rica

http://www.una.ac.cr/educare

educare@una.cr

En algunos de los planes de educación a las familias (P.Ed.F.) este tema fue trabajado mediante la estrategia "Tejiendo nuestro origen"; con base en el conocimiento previo de por qué tenemos nuestro nombre, analizamos las conexiones dentro de nuestro grupo familiar. Una docente del MEP expresó:

Me ha ayudado a tener una visión abierta, entendiendo a la familia como un sistema, en el cual las partes deben trabajar en conjunto y a la vez respetar las decisiones de los demás como seres únicos. (docente del MEP)

\section{Los sistemas familiares viven el cambio constante}

Las familias, producto de su evolución (epigénesis), atraviesan cambios constantes, entre ellos las transformaciones vitales de sus miembros (tareas y necesidades) que afectan y varían la interacción familiar del sistema total. Hernández Córdoba (1998) señala que la visión ecosistémica plantea una relación recíproca y complementaria entre generaciones, donde los ciclos de vida individuales son los "hilos con los que se teje el contexto familiar, el cual a su vez les da forma a los cambios del curso de la vida individual" (p. 45).

El modelo presentado en el artículo centró su interés en las familias con hijos o hijas pre y escolares; momento en que los sistemas deben cumplir para su autoorganización y mantenimiento, por ejemplo: la adaptación a un nuevo sistema como lo es la escuela.

Las estrategias metodológicas utilizadas en los P.Ed.F. se enfocaron en el reconocimiento de los diversos cambios vividos y su influencia en las interacciones familiares. Por ejemplo, se realizó un collage donde se mostraron bodas, nacimientos, muertes, divorcios, graduaciones y cambios laborales entre otros eventos importantes. Al realimentar, una maestra (CEN-CINAI) expresó: cada miembro de la familia sufre sus propios cambios que afectan una forma $u$ otra a los demás, lo cual siempre sirve para el crecimiento de toda la familia.

\section{La adaptación es un recurso de supervivencia}

La presencia del cambio en las familias implica adaptarse para mantener su homeostasis; lo que genera estrés y dificultades que forman parte de la cotidianidad, pero dependiendo de los recursos que el sistema tenga, su afrontamiento puede ser beneficioso o perjudicial para sus miembros.

Como estrategias metodológicas fueron utilizadas actividades que rescataran cambios que la familia ha atravesado y las diversas formas de cómo se han enfrentado, reforzando la capacidad de adaptación que demuestran. 


\section{Pertenencia, orden y equilibrio como órdenes de vida familiar}

Estos principios representan el método de constelaciones, en el cual se pueden observar conexiones y vinculaciones que están presentes y afectan las relaciones familiares.

Pertenecer significa"ser parte”y es un derecho fundamental."Desde la pertenencia se agrupa a una familia o cualquier sistema más allá de cualquier consideración moral, socioeconómica y política" (Vallejo Valencia, 2008 p. 151). Supone una obligación por no excluir a nadie de la red familiar; la cual incluye a miembros de la familia extensa, por consanguinidad o no; son todas aquellas personas que de alguna manera están vinculadas a esa "comunidad de destino".

El orden implica respetar la jerarquía de cada miembro; de esta forma, se reconoce el lugar de los que están primero sobre los que llegan posteriormente. "Los que ostentan una jerarquía más alta en el sistema familiar ... deben reconocer a los que estuvieron antes porque les aporta muchos beneficios, al poderse apoyar en dicha experiencia" (Súñer, 2015, - Quien lleva más tiempo tiene prioridad, párr. 1). Ostentar un lugar alto en las jerarquías implica ciertos beneficios, pero también obligaciones. Las estrategias metodológicas ejecutadas buscaron recordar personas de otras generaciones que, al existir, permitieron la vivencia de las generaciones actuales.

El equilibrio es una búsqueda natural de la homeostasis del sistema, el cual es recurrente en los intercambios humanos. Está presente el "dar y tomar", creador de vínculos mutuos. "Cuando no existe en una familia ... un equilibrio entre lo que sus miembros dan y lo que toman entonces se suscita el descontento y los sentimientos de culpa, lo que puede llevar a tener que compensar dichos desequilibrios" (Súñer, 2015, - Dar y tomar, párr. 1). Considerar estos principios de las constelaciones familiares puede aportar valiosa información a la comprensión de la dinámica del sistema.

\section{Los sistemas familiares asumen modelos de interacción y comunicación}

La formación de la dinámica familiar se ve influida por varios factores, uno de ellos es la comunicación. Para poder comprenderla, se toman como base los axiomas de comunicación propuestos por Watzlawick et al. (1995), de los que se destaca: la imposibilidad de no comunicarse, así como, considerar que, en la comunicación, además del contenido que transmite, hay un nivel relacional que influye la forma en que el contenido es recibido.

Otro factor que influye en la dinámica familiar es el manejo del poder, específicamente en la interacción sujetos progenitores y prole. Este se define como la capacidad de influencia de una persona en otra. Idealmente, entendido como liderazgo; debe estar en manos de sujetos progenitores o personas adultas de la familia, quienes guían la vida de su prole. Sin embargo, el manejo del poder se comparte entre sus miembros de acuerdo con la etapa de su descendencia. 
http://doi.org/10.15359/ree.24-2.9

ROR: https://ror.org/01t466c14 Universidad Nacional, Costa Rica

http://www.una.ac.cr/educare

educare@una.cr

Por su parte, Nardone et al. (2003) mencionan que las familias usan diferentes modelos de organización, los cuales se diferencian en tres aspectos fundamentales: sus reglas (sintaxis), los significados que surgen de la aplicación de esas reglas (semántica) y las acciones y comportamientos que se originan a raíz de las dos anteriores (pragmática). De la combinación de estos aspectos surge un manejo particular del poder en la familia y es representado por seis modelos: hiperprotector, democrático-permisivo, sacrificante, delegante y autoritario.

El modelo de interacción que una familia experimenta tiene su incidencia en el grado de cohesión familiar; factor importante de la dinámica que se observa mediante el vínculo emocional de los miembros de la familia entre sí y el nivel de autonomía individual que el sistema le permite tener a cada miembro. Es propicio que las familias tengan un vínculo que favorezca una cohesión caracterizada por cercanía, compromiso, tiempo compartido, seguridad, pero también debe ser compatible con la necesaria individualización y autonomía.

Para la exploración de la dinámica se utilizaron diversas estrategias, por ejemplo, la observación y registro de patrones de comunicación en el contexto familiar, luego eran analizadas sus repercusiones, según modelos de crianza identificados.

Considerados los postulados teórico-metodológicos y la generación del proceso, se prosiguió como correspondía a elaborar los planeamientos que fueran congruentes con el enfoque e hicieran viable la ejecución de los P.Ed.F.

\section{Planeamiento circular}

La ejecución del proceso de orientación familiar (Figura 3) se dio mediante un planeamiento circular que fuera coherente al enfoque sistémico utilizado. Con este propósito, el POF creó un esquema para su presentación (Figura 4) en el cual se pretendieron reflejar los principios de circularidad, entendida como interactividad entre las partes y permeabilidad, pues permite la posibilidad de realizar cambios (Valdés Cuervo, 2007). En este sentido se comprendió el plan como un todo, un sistema en el cual sus elementos se influyen recíprocamente, de modo que el cambio en una de sus partes se refleja en las otras y, a su vez, estas afectan su elemento original (O'Connor y McDermott, 1998). Esta idea refleja el pensamiento circular.

Específicamente en los proyectos de educación a las familias se realizó un planeamiento circular por cada sesión de trabajo; al menos fueron realizadas seis por institución o centro. Los elementos considerados en los planeamientos circulares fueron:

1. Meta de la sesión: es el propósito específico de la sesión, es muy concreta y clara, viable de lograr y se redacta preferiblemente utilizando verbos más interaccionales que impliquen una acción, por ejemplo; representar, compartir, establecer, relacionar, reencontrar, construir. 
http://doi.org/10.15359/ree.24-2.9

ROR: https://ror.org/01t466c14 Universidad Nacional, Costa Rica

2. Elementos teóricos de la sesión: son aquellos aspectos de la teoría (contenidos) en el área familiar que se planea trabajar.

3. Apoyo bibliográfico: indica los acompañantes teóricos sobre el tema específico a trabajar.

4. Metodología: es vivencial, de autorreflexión, grupal y de construcción.

5. Ejercicio grupal sistémico: son las estrategias que se ejecutarán para el cumplimiento de la meta. En la etapa productiva del desarrollo de la sesión se sugiere utilizar preguntas circulares (de visión más sistémica) que no se refieran al porqué de una situación o interacción, mejor sustituirlas por aquellas que "se refieren al cómo, cuándo, dónde".

6. Tarea: actividades fuera de la sesión que se solicita se realicen de una sesión a otra, por ejemplo, para identificar vínculos, observar interacciones, reconocer aportes de las generaciones anteriores, tomar decisiones para promover cambio o mantener lo existente y determinar fortalezas, en función del cumplimiento de la meta.

7. Retroalimentación: actividad cuyo fin es valorar los resultados de la sesión y tomar decisiones para implementar mejoras o pertinencia de próximas metas; aquí se integra lo vivenciado, unido al referente teórico que apoye y mejore el funcionamiento familiar.

Figura 4: Planeamiento circular.

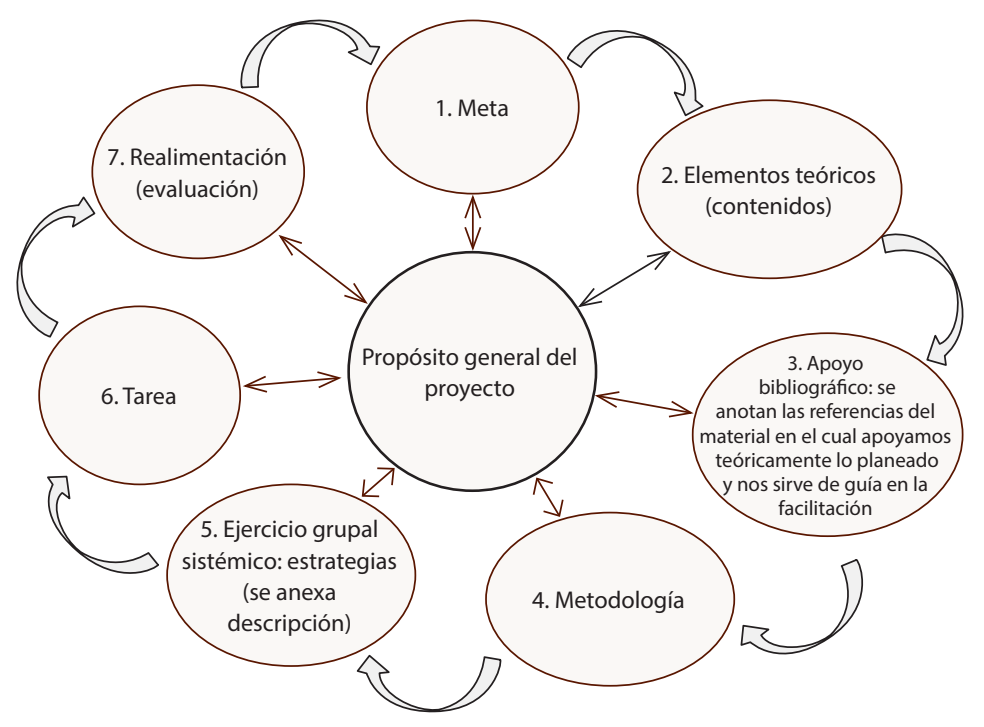

Nota: Elaboración propia. 
http://doi.org/10.15359/ree.24-2.9

ROR: https://ror.org/01t466c14 Universidad Nacional, Costa Rica

http://www.una.ac.cr/educare

educare@una.cr

En este modelo, posterior a cada sesión se solicitó, a quienes facilitaron, realizar una crónica de lo sucedido para rescatar algunos resultados en cuanto a reacciones de las personas participantes, evidencias del uso del enfoque sistémico, de la metodología y del desempeño del rol profesional.

Al planificar en este modelo, es importante tener claro que la metodología utilizada considera que las familias tienen su propio potencial para adaptarse y cambiar. Se confía en la capacidad de los sistemas familiares para equilibrar la forma en que funcionan, basándose en su historia y sus creencias. Por lo que no se trata de brindar soluciones o estrategias para que madres y padres resuelvan lo que podrían considerar como problemas; sino de facilitar oportunidades de construir o reconstruir sus propias interacciones. Además, cuando se planea la intervención familiar, se debe considerar que el proyecto con familias es un proceso en su totalidad y cada sesión en sí misma es un proceso. Por ello cada una debe trabajarse como si fuera la única vez en que participan padres, madres $u$ otras personas encargadas de las familias. Es posible, por ejemplo, que una madre se presente solamente una vez, varias veces o la totalidad de sesiones programadas; sin embargo, cada equipo de trabajo a cargo debe tomar la decisión de permitir o no esta serie de posibilidades.

Las sesiones que se vivencian tienen sentido por sí mismas. Cada sesión se debe trabajar desde la vivencia de cada familia, el referente teórico es un apoyo y las actividades o estrategias son un medio; lo importante es la vivencia (experiencias cotidianas) de cada participante y la oportunidad que tenga para realizar cambios en su dinámica familiar.

\section{Rol facilitador}

Un aspecto importante de retomar es el rol de quienes facilitan; su desempeño debe fortalecer las características sistémicas del proceso, visibilizando las conexiones entre acontecimientos, personas, sentimientos, entre otros. Como lo dice el principio de aprendizfacilitar, debe tener presente que no es "una persona experta" sino que su tarea radica en facilitar una intervención que genere cambios, teniendo claridad que el eje del trabajo son las vivencias de las personas participantes; que no se trata de "ayudar" sino de crear contextos para el cambio donde cada persona pueda realizar las reflexiones que requiera para comprender su dinámica familiar y decida hacer los ajustes necesarios para mejorar sus interrelaciones. Además, es importante considerar que el trabajo en grupos es un recurso significativo de aprendizaje y apoyo. Este medio ha sido catalogado por parte de Sanz Oro (2001, p. 111) como "una experiencia educativa única", donde sus participantes trabajan conjuntamente, rasgo que propicia relaciones de ayuda en la dinámica. Este se convierte en un espacio exclusivo para compartir las experiencias de crianza, tanto de la familia de origen como en la de procreación. La vivencia de exponer las propias experiencias junto a profesionales y progenitores ha sido de gran valor pues posibilita mayores enseñanzas y potencia el cambio debido a que el eje de 
trabajo lo constituyen las vivencias de las personas participantes; oportunidad, en opinión de Sanz Oro (2001), para reconstruir conductas y sentimientos asociados a problemas específicos.

Hay que destacar, además, que el trabajar con varios integrantes de las familias a la vez permite ampliar la cobertura que, por lo general, en otras modalidades es limitada, porque las iniciativas institucionales y comunales cuentan con pocos recursos económicos y humanos.

Como ya se ha mencionado, el referente teórico no es la base de las sesiones, sino un apoyo importante; no se enfoca en la exposición de la teoría, pero sí se evidencia en la dinámica de grupo. De allí que es importante que quien facilita tenga un fundamento sólido tanto de familia como del enfoque sistémico, que se refleje en su práctica.

\section{Implementación del modelo}

El modelo metodológico, a cargo de profesionales en orientación, se ha venido configurando a través de diversas experiencias en el POF (Calderón Monge et al., 2009; Villarreal Montoya et al., 2014) y consolidado en las últimas iniciativas de capacitación, entre los años 2013 y 2015, que surgieron con el fin de ampliar la formación teórico-metodológica en profesionales que laboraban en las escuelas: San Jerónimo, Las Gravilias, JoséTrinidad Mora, Concepción, Finca La Caja, Hospital de Niños (Centro de Apoyo en Pedagogía Hospitalaria, CEAPH), además los Centros de Atención Institucional del Ministerio de Justicia y Paz (CAI), San Rafael y La Reforma. Todas las anteriores ubicadas en Heredia, Alajuela y San José, a estas instancias se sumaron oficinas locales del CEN-CINAI situadas en todas las provincias del país (Osa, Siquirres, Sarapiquí, Los Sitios de Moravia, Santa Cruz de Guanacaste, Turrialba, Oreamuno de Cartago, región sur de San José, Nicoya, Pérez Zeledón, Curridabat, Pococí, Jicaral, Buenos Aires y Puntarenas centro).

Se trabajó con el fin de que las personas participantes fortalecieran sus habilidades de facilitación basadas en criterios sistémicos y se diera sentido y pertinencia a la planificación de acciones dirigidas a mejorar la calidad de relaciones en la vida de las familias que conformaban los diferentes centros e instituciones. Se pretendió proveer de herramientas conceptuales y prácticas, a funcionarios y funcionarias de las áreas de nutrición, psicología, terapia de lenguaje, formación docente en primaria y preescolar, educación especial, orientación, psicología, trabajo social, administración educativa, problemas emocionales y conducta, y psicopedagogía, para que implementaran proyectos dirigidos a madres, padres, adultos y adultas responsables de niños y niñas, que según su ubicación se encontraban inmersos en contextos vulnerables de pobreza.

Las capacitaciones efectuadas tomaron la forma de cursos, denominados: "Creciendo en familia"y"Fortaleciendo familias en prácticas de parentaje". En total participaron 59 profesionales, quienes, organizadas en equipos de trabajo logran ejecutar un total de 26 planes de educación a las familias. 
http://doi.org/10.15359/ree.24-2.9

ROR: https://ror.org/01t466c14 Universidad Nacional, Costa Rica

http://www.una.ac.cr/educare

educare@una.cr

\section{Ejes temáticos transversales}

- Bases epistemológicas sobre el posicionamiento sistémico.

- Principios teóricos sobre la teoría sistémica familiar.

- Construcción de patrones relacionales en el sistema familiar.

- Metodología sistémica para orientar a padres y madres de familia.

- Facilitación a partir del respeto y la cosmovisión relacional

\section{Proceso de orientación familiar vivenciado (Figura 5)}

\section{IETAPA: Una mirada sistémica de la convivencia familiar}

A. La familia como sistema

- Visión interaccional de la familia (del ser individual al grupo familiar).

- Pertenencia e historia de la familia

- El cambio en la familia: etapas del desarrollo.

- Revisión metodológica de las estrategias sistémicas de la etapa.

B. Estructura familiar

- Sistema y subsistemas

- Principios sistémicos: Orden, equilibrio y pertenencia.

- Revisión metodológica de las estrategias sistémicas de la etapa.

C. Dinámica familiar

- Comunicación

- Poder

- Cohesión (Límites, vínculos afectivos y reglas).

\section{ETAPA: Acercamientos al planeamiento de intervención sistémica}

A. Metodología sistémica

- Planificación, estrategias y técnicas.

- Formulación de proyectos 
http://doi.org/10.15359/ree.24-2.9

ROR: https://ror.org/01t466c14 Universidad Nacional, Costa Rica

http://www.una.ac.cr/educare

\section{ETAPA: Realimentación de las experiencias y valoración de los cambios promovidos}

Es importante anotar que la realimentación fue constante durante las capacitaciones. Esto significó que el equipo capacitador, con base en el proceso de orientación familiar, fue tomando decisiones respecto a la planificación y desarrollo de cada una de las sesiones, para valorar los alcances obtenidos, las limitaciones y la pertinencia de las estrategias en relación con su coherencia con el enfoque sistémico. A partir de ello, se revisan los planeamientos y se realimentan para hacer las adaptaciones necesarias.

Figura 5: Proceso de orientación familiar vivenciado

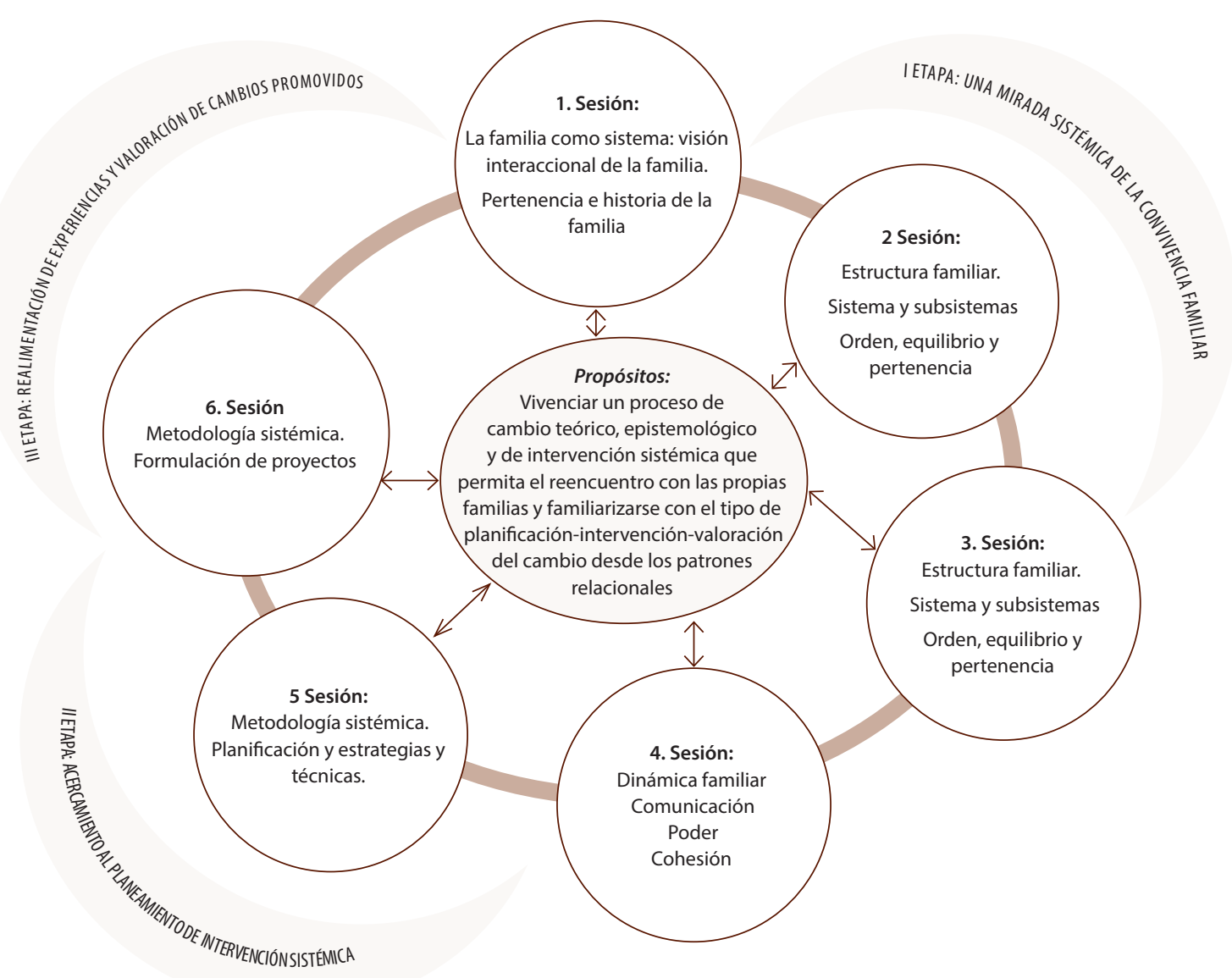

Nota: Elaboración propia. 
http://doi.org/10.15359/ree.24-2.9

ROR: https://ror.org/01t466c14 Universidad Nacional, Costa Rica

http://www.una.ac.cr/educare

educare@una.cr

\section{Organización de las capacitaciones}

Cada una de las capacitaciones se organizó en dos partes que se describen a continuación:

\section{Fase}

En esta primera parte, se estudiaron los fundamentos teórico-metodológicos de la perspectiva sistémica para trabajar con padres, madres y otras personas encargadas de sus familias. Las personas participantes asistieron en promedio a 12 sesiones de capacitación, durante las cuales aplicaron sus conocimientos, mediante la planificación de un proyecto llamado "Plan de trabajo de educación a la familia" (que se ejecutó en la segunda parte la capacitación), dirigido a la población de familias del lugar de trabajo de los equipos interdisciplinarios capacitados.

Durante la primera parte del curso, el grupo tuvo la oportunidad de vivenciar la metodología sistémica en el proceso de comprensión de su propia familia; asimismo, se experimentaron los principios teóricos y metodológicos mencionados, por ejemplo, realizar y ejecutar un planeamiento circular sistémico con grupos familiares en sus comunidades. En el marco del posicionamiento sistémico y desde el quehacer del proyecto fue indispensable la visualización de las familias de las cuales son parte quienes participaron y sobre las que se consideró serían las primeras beneficiadas en este proceso. Aun cuando las personas capacitadas fueran solteras o vivieran solas, esto no fue un impedimento para vivenciar el proceso, porque independientemente de estas características, forman parte de una familia.

\section{Fase}

En esta fase del curso, cada equipo de trabajo ejecuta su proyecto de educación a las familias con una duración de seis sesiones cada uno. Acontecida la segunda parte del curso, los equipos interdisciplinarios ejecutaron, evaluaron y sistematizaron el plan mencionado. Las personas capacitadas fueron 59, quienes realizaron en total 26 planes de educación a las familias en sus respectivas comunidades; en estas iniciativas participaron y fueron beneficiadas 345 personas y sus familias.

Las experiencias adquiridas fueron compartidas en un evento público de divulgación de los resultados en el cual participaron representantes de las familias, personal facilitador, estudiantes de la carrera de Orientación, directoras y directores de los centros y autoridades de las instituciones participantes.

\section{Realimentación}

Con el fin de evaluar los resultados del proyecto, se utilizó la modalidad de evaluación de procesos, que se inicia con una etapa de planificación, definición de estrategias para medir el cumplimiento de metas, valoración de estas, así como de las limitaciones presentadas. Como 
http://doi.org/10.15359/ree.24-2.9

ROR: https://ror.org/01t466c14 Universidad Nacional, Costa Rica http://www.una.ac.cr/educare

educare@una.cr

insumo, se utilizaron los informes de avance y finales realizados por quienes participaron, así como las crónicas, videos, fotografías, evaluaciones orales de cada una de las sesiones ejecutadas.

\section{Resultados de la experiencia}

Como resultados significativos de este modelo metodológico de capacitación para personas facilitadoras de proyectos dirigidos a familias destacan: el reencuentro con la propia familia, mayor conocimiento sobre la dinámica familiar, generar y desarrollar proyectos propios, efectuar una intervención más relacional que permitiera facilitar para el cambio. Es importante mencionar que 59 personas participaron de esta capacitación.

Algunos resultados obtenidos respecto a ver a sus propias familias como un sistema relacional se detallan a continuación, con el apoyo de algunas citas etnográficas directas de las personas facilitadoras que participaron. Estas responden al primer propósito específico.

\section{Ver a la familia como un sistema}

De acuerdo con el enfoque conceptual utilizado, las familias son un sistema de interacción donde la relación entre sus miembros determina su funcionamiento (Espinal et al., 2004-2006). La comprensión de esta idea es base para poder cambiar la visión lineal del grupo familiar, a una visión circular, en la que se concibe a la familia como un todo, donde lo que cada persona hace o deja de hacer afecta al resto y viceversa. La comprensión de esta idea era importante para lograr metas de la capacitación, lo cual fue evidente en los comentarios que algunas personas hicieron, después de involucrarse y observar sus propias familias:

Reformar las memorias, evocando y sopesando el camino recorrido; desde la familia de origen y su influjo en la familia actual. Me ha permitido estar tomando consciencia de las pautas relacionales y me he propuesto hacer algunos cambios y mantener otros. (Docente del Ministerio de Educación)

Ha servido bastante para analizar mi vida e implementar estrategias para mejorar el vínculo familiar. (Maestra en terapia de lenguaje-CENCINAI)

Como se pudo identificar, un propósito fundamental de la visión sistémica, asumido también en las experiencias realizadas, fue reconceptualizar a las familias desde la convivencia relacional y de la construcción de vínculos.

\section{Reencuentro con sus propias familias}

El planeamiento y las estrategias escogidas por el equipo capacitador tenían como propósito que cada persona tomara como base su propia experiencia vital familiar para el desarrollo de los 
http://doi.org/10.15359/ree.24-2.9

ROR: https://ror.org/01t466c14 Universidad Nacional, Costa Rica

http://www.una.ac.cr/educare

educare@una.cr

talleres. Esto fue un logro significativo, pues también influyó en que la asistencia de profesionales participantes fuera constante, además se consiguió que tuvieran un reencuentro con sus propias familias y la diversidad familiar. Con este fin, se buscaba que vivenciaran un proceso de facilitación sistémica desde su experiencia como personas integrantes de un sistema familiar, ya que la pertenencia es un principio básico (Vallejo Valencia, 2008); para que desde allí pudiesen incursionar al cambio de paradigma en sus prácticas profesionales. Al principio, las personas participantes venían con la actitud de solo recibir conocimientos, pero cuando se empezó a trabajar a partir de sus experiencias de vida, algunas mostraron resistencia, refugiándose detrás de un rol profesional, pero conforme fueron avanzando las sesiones, se logró que rompieran esa barrera y se permitieran trabajar desde una posición personal, cuestionando cómo eran sus propias familias en realidad. Una participante comentó:

Yo venía como siempre a los cursos, con mi cuaderno y mi lapicero, no creí que íbamos a trabajar con nosotros mismos. (Maestra de Guanacaste)

Otra participante, luego de las primeras sesiones en las que se favorecía la observación sobre su propia convivencia familiar expresó:

Yo me pensé... yo no vuelvo...pero aquí estoy, contenta. (Maestra de Guanacaste, CENCINAI)

\section{Mayor conocimiento de la dinámica familiar}

Durante las sesiones se trabajó sobre diferentes aspectos que influían en la dinámica familiar, tales como la comunicación, cambios a través del ciclo vital, sus tareas y crisis del desarrollo propias (Hernández Córdoba, 1998), también los diferentes modelos de crianza usados, las cercanías o distancias entre los miembros de las familias, así como la importancia del afecto (Nardone et al., 2003). Cada uno de estos elementos teóricos se abordó en las sesiones mediante estrategias que posibilitaran a cada persona volverse hacia el interior de su grupo familiar y hacer una reflexión respecto a su funcionamiento. Por supuesto que, como parte de la metodología empleada, la facilitación sistémica provee del espacio y la oportunidad para que las personas realicen los cambios que requieran -si lo desean- en busca de relaciones familiares más saludables; pero, a la vez, es respetuosa del ritmo que cada quien necesita para realizar mejoras en su dinámica. Una expresión respecto al trabajo personal en la construcción de estos vínculos es:

He comprendido desde la perspectiva (sistémica) ciertos comportamientos de mis hermanos, retomar patrones de crianza de mi familia y mejorar como persona y cambiar actitudes que pueden perjudicar a nivel familiar. (Psicólogo del CEN-CINAI) 
http://doi.org/10.15359/ree.24-2.9

ROR: https://ror.org/01t466c14 Universidad Nacional, Costa Rica http://www.una.ac.cr/educare

educare@una.cr

\section{Metodología más relacional}

Para el segundo propósito: construir un planeamiento de intervención familiar sistémica, se lograron elaborar 26 planes de educación a las familias; concebidos desde los principios sistémicos de totalidad y circularidad que posibilitan el cambio (O'Connor y McDermott, 1998; Valdés Cuervo, 2007).

Un tema transversal durante toda la capacitación fue el de acercar a quienes participaron a la metodología sistémica. Familiarizarse con el tipo de planificación -intervención, valoración del cambio desde los patrones relacionales y convertirse en una persona facilitadora del cambio fueron algunos retos propuestos para esta actividad formativa. Para mediar su logro, se ejecutaron variadas estrategias, por ejemplo, previo a cada sesión, se brindaba material bibliográfico sobre el tema eje a vivenciar, para que la persona pudiese tener un marco referencial teórico, con base en el que se les pedía realizar algunas tareas con sus familias. Por otro lado, las sesiones eran planeadas con perspectiva sistémica (planeamiento circular) que brindara a sus participantes el modelaje práctico que les diera mayores recursos para el desarrollo posterior del plan de educación a las familias en sus centros de trabajo. Algunas manifestaciones respecto a la vivencia son:

Me ha gustado la metodología y visión propuesta, considero que es una oportunidad no solo para capacitarnos sino para sensibilizarnos respecto a las familias que atendemos. (Nutricionista del CEN-CINAI)

Es más provechoso ya que se trabaja con otra visión, entendiendo el proceder de las personas, ampliar problemas con el trabajo con familias, mejorando el entendimiento de cada dinámica. (Socióloga del Ministerio de Educación)

\section{Elaboración y seguimiento de sus propios proyectos: Plan de educación a las familias}

En este apartado se puntualizan logros correspondientes para el tercer objetivo específico. Durante la capacitación se fue construyendo una propuesta de intervención educativa dirigida a madres, padres o personas encargadas que cada equipo de profesionales realizaría en su lugar de trabajo. Una vez presentada y hechos los planeamientos circulares, se abrió el espacio para socializarlos, corroborar que siguieran la metodología sistémica y hasta se logró ensayar una sesión en vivo, entre pares, de las distintas organizaciones e instituciones. Con ello se pretendió practicar, facilitar el modelaje y retroalimentar la vivencia, enriqueciendo con sugerencias aspectos que se consideraban factibles de mejora en relación con la coherencia teórico-práctica. Esta estrategia, enriquecería la experiencia de trabajar con las familias, desde una perspectiva sistémica. 
http://doi.org/10.15359/ree.24-2.9

ROR: https://ror.org/01t466c14 Universidad Nacional, Costa Rica

http://www.una.ac.cr/educare

educare@una.cr

Para las personas participantes, al principio fue un reto que les preocupaba, sobre todo porque en el grupo había diversidad en cuanto a formación profesional y algunas personas alegaban que estaban capacitadas para trabajar con niños y niñas, pero no con las personas adultas de la familia. Aún, por parte de las personas profesionales de ayuda, había una tendencia a planear desde la experiencia lineal, donde hay una persona experta y otras que se nutren de ella. Sin embargo, en una visión sistémica, quien planea intervenciones con familias tiene la claridad de que estas poseen los recursos necesarios para edificar su dinámica y salir adelante con sus retos, por lo que su papel es el facilitar para que la familia construya una relación saludable que permita el desarrollo de cada integrante que la compone y no la de un sujeto experto que brinda soluciones. Si quien facilita tiene esta claridad, su metodología de trabajo lo refleja, entonces no intervendrá con "charlas" o exposiciones, sino que generará oportunidades de interacción que permitan a las familias trabajar sobre su propia experiencia, retroalimentarse con la de las otras familias y adaptarse al continuo cambio por el que atraviesa y del que, a veces, no es consciente. En este proceso, tanto el modelaje de la metodología sistémica, ejecutado en cada sesión y facilitado por el equipo capacitador, así como contar con acompañamiento continuo, en el planeamiento de sus proyectos locales, fue apreciado. Algunas reacciones al respecto expresan:

Mehagustado muchoporque através de lavivenciaseadquierenyentiendenlos conocimientos que luego se llevan a la práctica en los diferentes establecimientos. (Maestra del CEN-CINAI)

Este tipo de curso es muy enriquecedor, siempre se aprenden cosas nuevas y experiencias que nos fortalecen como persona en todos los ámbitos, lo cual nos sirve como herramienta de trabajo y crecimiento personal. (Orientadora del Ministerio de Educación)

Una vez que el grupo facilitador se encontró en la ejecución de sus proyectos con familias, pudo observar que había mucho interés de los padres y madres de participar porque se trabajaba sobre sus mismas situaciones, no sobre casos ajenos o sobre recomendaciones que no sabían cómo aplicarlas a sus propias familias, sino con sus propias dificultades, inquietudes y también fortalezas. localidad:

Una facilitadora describe su percepción posterior a la primera ejecución del taller en su

Tienen una emoción tan grande de hablar de sus familias y (es que) no tenían esa oportunidad antes. (Orientadora del Ministerio de Educación)

Me llamó mucho la atención cómo ellas mismas iban desarrollando el tema y llevando la pauta. (Directora de establecimiento del CEN-CINAI) 
http://doi.org/10.15359/ree.24-2.9

ROR: https://ror.org/01t466c14 Universidad Nacional, Costa Rica http://www.una.ac.cr/educare

El modelo de intervención sustentado en principios sistémicos que fue ejecutado brindó mayor seguridad a sus facilitadores en el trabajo con los grupos familiares. Se tuvo claro que no era un requisito saber mucho de resolución de conflictos, porque no estaban en la obligación de resolver, sino de facilitar. Sí era necesario contar con la claridad epistemológica sistémica que fundamentaba la práctica, es decir, que comprendieran que la familia es única, que es un sistema por lo que la interacción es importante de observar y que lo que sucede a su interior no es responsabilidad de una sola persona, sino de la interacción entre sus miembros, y que el cambio es una característica constante. Una participante manifestó respecto a su aprendizaje en la capacitación:

Una experiencia enriquecedora a nivel profesional que te instrumentaliza, dándote una perspectiva diferente para el abordaje a las familias (Maestra de preescolar, Ministerio de Educación).

\section{Facilitar para el cambio}

El cambio en la familia es una característica que la va llevando a atravesar diversas etapas en su desarrollo: niñez, adolescencia, adultez, pero como en este recorrido el desequilibrio está presente, ocasiona que la familia se desestabilice y se sumerja en dificultades relacionales (Hernández Córdoba, 1998). Entender que las familias requieren aprender a flexibilizarse para adaptarse a los cambios es una tarea de la facilitación.

Cuando se les preguntó a las personas participantes sobre qué significa facilitar para el cambio, una persona agregó:

(Es) ser guía en el proceso sin necesidad de dirigir a las personas para el cambio, dejando que ellos mismos identifiquen fortalezas y debilidades y sean quienes busquen soluciones, se movilicen al cambio. (Trabajadora social del Ministerio de Justicia y Paz)

Esto denota un respeto por la propia familia y a la vez un compromiso de favorecer su desarrollo. Para otras profesionales, su aprendizaje apoya la labor que ya venía realizando:

Ya yo tenía bases de la importancia de partir de la experiencia de las personas, el rol de facilitadora y la riqueza de las técnicas para recuperar desde lo vivencial pero el curso amplió mucho más la visión, sobre todo la riqueza de lo no verbal y la capacidad de los sistemas, de autorregularse, el poder de la historia y de los procesos no causa estrés que se jueguen las relaciones. (Psicóloga) 
http://doi.org/10.15359/ree.24-2.9

ROR: https://ror.org/01t466c14 Universidad Nacional, Costa Rica

http://www.una.ac.cr/educare

educare@una.cr

Al principio, algunas docentes se sentían temerosas de su participación, frecuentemente decían:

Tuve que volver a leer la lectura de hoy porque no entendía...nunca me habían hablado de eso. (Maestra)

Pero en su avance con las lecturas, tareas realizadas sobre su propia interacción familiar y la ejecución del taller, su discurso fue más empoderado, porque durante las sesiones de capacitación comprendieron que, independientemente de la formación profesional, el hecho de que todos y todas formamos parte de una familia brinda una valiosa experiencia en ese campo.

Lindo curso, tiene sus elementos muy interesantes que no había descubierto en el pasado en relación al acompañamiento a familias. (Docente)

Otra participante añadió:

La actividad fue muy enriquecedora, tanto que permitió a través de situaciones concreta ampliar con mayor sencillez algunos términos que a veces se dificultan, pero que son parte de la cotidianidad desde esta perspectiva. (Docente)

\section{Involucramiento de infantes en las experiencias}

Los planes de educación a las familias fueron pensados para ejecutarlos con los sujetos progenitores de los niños y niñas, aunque algunos proyectos locales incluyeron la participación de infantes en los talleres. Esto, como estrategia de favorecer la participación de las madres, quienes muchas veces no podían asistir a este tipo de actividades, porque no tienen dónde dejarles. Además, las actividades favorecieron la relación entre las madres participantes y sus hijos e hijas. Una facilitadora expresó:

Hemos integrado mucho a los niños, eso ha funcionado para que asistan. (Nutricionista)

Esta iniciativa de involucrar conscientemente a los hijos e hijas, en los talleres a las familias, trajo muchos beneficios para esta niñez. La mayoría de las funcionarias y funcionarios capacitados coinciden en que los talleres favorecen a las personas participantes porque una familia saludable es un ambiente más oportuno para el desarrollo integral, además unos progenitores con mayor claridad de su rol, propiciarán una convivencia más nutricia a sus niños y niñas. Algunos comentarios al respecto son:

Padres y madres con mayor educación para el ejercicio del rol que redunda en que los niños y las niñas son tratados y educados de la mejor manera sin violencia y con respeto. (Trabajadora social del Ministerio de Justicia y Paz) 
http://doi.org/10.15359/ree.24-2.9

ROR: https://ror.org/01t466c14 Universidad Nacional, Costa Rica http://www.una.ac.cr/educare

educare@una.cr

Espero que las familias los respeten mucho más (a los niños y niñas), les den su lugar con voz y voto de acuerdo a sus posibilidades. Además, que el problema no se achaque solo al menor, sino que es familiar. (Maestra del CEN-CINAI)

\section{Resultados reportados por las personas facilitadoras de su trabajo con las familias}

Los resultados propios del trabajo directo con padres y madres en las comunidades presentados en los informes de las facilitadoras capacitadas son una prueba directa del impacto de la experiencia realizada. En total se beneficiaron 345 personas, quienes como parte de un sistema familiar influyen recíprocamente en las demás personas miembros. De modo que el cambio en una de sus partes se refleja en las otras (O'Connor y McDermott, 1998).

Precisamente, porque se iba trabajando sobre la experiencia vital de cada participante, esto va influyendo directamente en la dinámica presente de las familias.

Hay una mamá que trataba mal a sus chiquitos, [ya] habíamos hablado con ella ... [y] no había cambios; [pero] no ha faltado a las sesiones y ya hemos visto cambios en sus reacciones con los chiquitos, ya no les grita cuando riegan el fresco. (Funcionaria del CEN-CINAI, citada en Monturiol, 2015, Parentaje respetuoso, párr. 2)

Con esto se remarca que un trabajo práctico donde las familias "elaboren sobre su propia realidad, tiene mayor efecto para mejorar conductas que el [solo] "discurso" [de] cómo mejorar las prácticas en el trato con los niños y niñas" (Villarreal-Montoya, et al., citadas en Monturiol, 2015, Parentaje respetuoso, párr. 3).

En general, la mayoría de las facilitadoras capacitadas muestra satisfacción por las experiencias vividas. Algunas de sus expresiones son:

Yo me siento muy privilegiada de participar en esta capacitación a pesar de que ya tenía una amplia experiencia profesional. (Psicóloga del CEN-CINAI)

Estas capacitaciones han tocado mi corazón, en el transcurso de mi vida he tenido situaciones de tristeza y alegría, a nivel personal y familiar y aquí he podido crecer como ser humano. A nivel como profesional me he sensibilizado aún más al tratar con las familias que atendemos. (Maestra del CEN-CINAI)

La orientación familiar, basada en un enfoque ecológico-sistémico, ve a las familias en una constante interacción de redes intra y extrasistémicas, por lo que los esfuerzos disciplinares se enfocaron en apoyar al sistema familiar para potenciar fortalezas y lograr mejorar las condiciones para afrontar el constante cambio. 
http://doi.org/10.15359/ree.24-2.9

ROR: https://ror.org/01t466c14 Universidad Nacional, Costa Rica

http://www.una.ac.cr/educare

educare@una.cr

\section{Conclusiones}

Concluida la investigación, se determina que haber implementado el modelo de intervención permitió fortalecer habilidades de facilitación en profesionales de diversas áreas; ello les brindó mayor seguridad en el trabajo con los grupos familiares, al crear espacios de oportunidad para que las personas puedan hacer cambios necesarios en busca de relaciones familiares nutridoras, si así lo quieren. Apoyar a las familias para que logren flexibilizarse ante las transformaciones que viven es orientar para facilitar el cambio.

De forma específica, el acercamiento teórico y epistemológico de las personas facilitadoras al enfoque sistémico les permitió visualizar a las familias como un sistema en interacción, que inicia por reencontrarse con sus propias familias y ver los vínculos que se establecen entre sus miembros y cómo estos se retroalimentan constantemente. Esto tuvo una influencia importante en la metodología utilizada en los planes de educación familiar ejecutados, porque, al igual que quienes participaron de ellos, sensibilizó a la persona capacitada en su rol facilitador, al verse como parte de un sistema familiar.

Teniendo claridad de los principios sistémicos, las personas facilitadoras pudieron construir los planeamientos para la intervención familiar, tomando en cuenta aspectos metodológicos propuestos por el modelo: un proceso de orientación por etapas, asumir un rol facilitador (aprendiz-facilitar, cambiar-facilitar, multiplicar-multiplicar) y un planeamiento circular, en donde todos los elementos son vinculantes.

Una vez asimilados los principios teóricos-metodológicos sistémicos y con un plan de educación familiar revisado y ensayado dentro de los grupos de capacitación, fue posible su implementación; se contó con una participación nutrida de las familias, atribuida a que la metodología permite a cada persona trabajar desde su propia experiencia familiar; por otro lado, la persona facilitadora tiene claro que no es su tarea resolver, sino facilitar, porque la familia tiene fortalezas que el proceso orientador le ayudará a reconocer.

El equipo capacitador, con su especificidad en un área disciplinar (orientación familiar), ha contribuido en la formación teórico-metodológica y consolidación de aprendizajes previos de equipos interdisciplinarios para desarrollar, con mayor pertinencia, intervenciones con las familias de sus comunidades, por lo que se recomienda su implementación en el trabajo con grupos familiares.

En el marco de madurez del POF surge el modelo metodológico presentado; su accionar estuvo permanentemente fundamentado en los principios del enfoque elegido y sobre él, la observación y la retroalimentación crítica del equipo capacitador como esfuerzos para mantener coherencia e integración entre la teoría y la práctica. 
http://doi.org/10.15359/ree.24-2.9

ROR: https://ror.org/01t466c14 Universidad Nacional, Costa Rica http://www.una.ac.cr/educare educare@una.cr

La intervención metodológica sistémica permite al profesional, ya sea en orientación familiar u otra profesión de ayuda, proveer a los sujetos progenitores una experiencia de aprendizaje que tenga una significación personal; que promueva oportunidades para trabajar en la transformación; que favorezca el cambio en el sistema familiar y, de esta forma, promueva mejores formas de convivencia social.

\section{Referencias}

Arias Gallegos, W. L. (2012). Algunas consideraciones sobre la familia y la crianza desde un enfoque sistémico. Revista Psicología Arequipa, 2(1), 32-46. https://goo.gl/bF2KVH

Bautista, N. P. (2011). Proceso de la investigación cualitativa. Epistemología, metodología y aplicaciones. El Manual Moderno.

Bisquerra Alzina, R. y Álvarez González, M. (2011). Los modelos de orientación. En R. Bisquerra Alzina (Coord.), Modelos de orientación e intervención psicopedagógica (pp. 55-65). Wolters Kluer.

Calderón Monge, E., Villalobos Cordero, A. L. y Villanueva Barbarán, R. (2009). Multiplicando esfuerzos para fortalecer la familia desde la orientación. Revista Electrónica Educare, 13(1), 89-102. https://doi.org/10.15359/ree.13-1.7

Espinal, l., Gimeno, A. y González, F. (2004-2006). El enfoque sistémico en los estudios sobre la familia. Revista Internacional de Sistemas, 14, 21-34.

Fuhrmann, I. y Chadwick, M. (1998). Fortalecer la familia. Manual para trabajar con padres. Editorial Andrés Bello.

Hernández Córdoba, Á. (1998). Familia, ciclo vital y psicoterapia sistémica breve. El Búho.

Instituto sobre Alcoholismo y Farmacodependencia. Área Desarrollo Institucional. Proceso de Investigación. (2011). Investigaciones sobre alcoholismo y farmacodependencia. Costa Rica, 2006-2010. Autor. http://www.iafa.go.cr/images/descargables/conocimiento/62.Investigaciones-Sobre-Alcoholismo-y-Farmacodependencia-Costa-Rica-2006-2010.pdf

Ministerio de Salud, Organización Panamericana de la Salud. (2004). La violencia social en Costa Rica. Autor. http://www.bvs.sa.cr/php/situacion/violencia.pdf

Monturiol, S. (Setiembre, 2015). Por un ambiente familiar sin violencia. Campus, 25, 268. http:// www.campus.una.ac.cr/2015setiembre pag03.html

Nardone, G., Giannotti, E. y Rocchi, E. (2003). Modelos de familia. Conocer y resolver los problemas entre padres e hijos. Herder. 
http://doi.org/10.15359/ree.24-2.9

ROR: https://ror.org/01t466c14 Universidad Nacional, Costa Rica

http://www.una.ac.cr/educare

educare@una.cr

O'Connor, J. y McDermott, I. (1998). Introducción al pensamiento sistémico. Recursos esenciales para la creatividad y la resolución de problemas. Urano.

Prieto Cruz, O. (2015) Familia y adolescencia en Costa Rica. El caso de las familias expulsoras. Revista Ciencias Sociales, 147, 59-72 Universidad de Costa Rica https://doi.org/10.15517/ rCs.v0i147.19793

Sanz Oro, R. (2001). Orientación psicopedagógica y calidad educativa. Pirámide Ediciones.

Sibaja, C. (2 de agosto de 2018). Violencia infantil: De epidemina a pandeminia. Semanario Universidad. Sección Diálogos sobre Bienestar. https://semanariouniversidad.com/ suplementos/violencia-infantil-de-epidemina-a-pandeminia/

Súñer, A. (2015). Constelaciones familiares. Terapia sistémica de enfoque fenomenológico. Bases teóricas. https://goo.gl/DFTvb3

Universidad de Costa Rica y United Nations Children's Fund. (2012). VII Estado de los derechos de la niñez y la adolescencia en Costa Rica. Autor.

Valdés Cuervo, Á. A. (2007). Familia y desarrollo: Intervenciones en terapia familiar. Manual Moderno.

Vallejo Valencia, M. (2008). Constelaciones familiares. Para liberar la energía del amor y de la vida. Aguilar.

Villagra, M. (Mayo, 2011). Epidemia de violencia infantil contagia al país. Campus Digital, Sección Actualidad. 22, 220. http://www.campus.una.ac.cr/ediciones/2011/mayo/2011mayo pag04.html

Villarreal Montoya, C., Villalobos Cordero, A. L. y Villanueva Barbarán, R. (2014). La modalidad de agentes multiplicadores para orientar a madres y padres de familia. Revista Electrónica Educare, 18(1), 219-238. http://doi.org/10.15359/ree.18-1.10

Watzlawick, P., Beavin Bavels, J. y Jackson, D. D. (1995). Teoría de la comunicación humana. Interacciones patologías y paradojas. Herder Editorial. 